\title{
AN INVESTIGATION OF MICROSTRUCTURAL EVOLUTION OF PM ALLOY N625 DURING INTERRUPTED HOT ISOSTATIC PRESSING (HIP) CYCLES
}

\author{
Brian A. Hann \\ Crucible Compaction Metals \\ Oakdale, PA 15071 \\ and \\ Ian Nettleship and Scott A. Schmidt \\ University of Pittsburgh \\ Pittsburgh, PA 15261
}

\begin{abstract}
PM Alloy N625 is used primarily in environments where high strength and corrosion resistance are required. The pre-alloyed powder is manufactured by inert gas atomization and consolidated by hot isostatic pressing (HIP). This study used dimensionless parameters to directly compare the microstructure evolution of this alloy with the evolution of the geometric models used for HIPing. The results suggest systematic differences between the microstructure evolution of the experiments and the model geometries.
\end{abstract}




\section{Introduction}

The production of superalloys by powder metallurgy offers potential advantages over casting. These include the elimination of macroscopic segregation and solidification structures as well as the potential benefit of near-net shape capability.(1) A variety of techniques have been used to consolidate superalloy powders. Hot isostatic pressing (HIPing) is one of the most effect techniques for removing all the pore volume. Given the expense of this technique, the prediction of the shape and size of the steel 'can' is a significant issue.

Other than direct experiment, the most popular approach to the prediction of shrinkage in HIPing is continuum mechanics modeling.(2) However, the intrinsic limitation of this approach is the series of experimental measurements that must to be performed on every material to determine the required constitutive parameters. An alternative approach is to use micromechanical models to predict the constitutive parameters needed to implement the continuum mechanics models. The micromechanical models are commonly based on the physical processes that are thought to control the kinetics of densification. Very simple ordered microstructures are commonly used to scale the physical processes to the microstructure. Hot isostatic pressing diagrams are an example of this type of model.(3) The diagrams compile the calculated densification kinetics based on knowledge of the densification mechanisms, a range of material parameters, and the isothermal conditions used for HIPing.

The ordered microstructures used in the micromechanical models have not changed much since they were first proposed for analytical models of sintering.(4)(5) The model for the initial stage of HIPing, sometimes referred to as stage 1, involves a microstructure made up of equisized spheres. The simplest modcls use ordered arrangements of spheres such as face centered cubic or simple cubic. The HIPing diagrams,(3) use a model based on dense random packing of spheres which allows for an increase in coordination number during densification.(6) The initial stage is used to a relative density of $92 \%$. The final stage model, sometimes referred to as stage 2 then takes over. This involves tetrakiadecahedon grains with spherical pores on the vertices.

In the recent past, the evolution of microstructures during sintering and HIPing has been compared with the evolution of the ordered geometries used to represent the stages of sintering.(7) This comparison used metric stereological properties which can be easily 
measured and readily calculated for the model stages. Surface area densities of solid-solid interface and solid-vapor interface were chosen for this purpose because they have unique values for any microstructure and they are commonly used to calculate derived microstructural quantities such as grain size and pore size.(8) The direct comparison of the models and the experiments, as a function of density, required the use of dimensionless parameters which avoided the need to define a microstructural length scale in either the experiments or the models.(7) The dimensionless parameters also allowed microstructures to be compared independent of issues relating to length scale.

In this study, the microstructure evolution during HIPing was compared with the evolution of the model stages as a function of density. The material was a nitrogen gas atomized 625 alloy powder. The final HIPed material combines high strength and good corrosion resistance.(9) For initial stage sintering both face centered cubic (fcc) and simple cubic (sc) arrangements of spheres were used in the model. The results for the model stages were plotted between their limits of self consistency. No physical reasoning was used to further limit the density ranges of the model stages.

Two dimensionless parameters were used for the model calculations. The first parameter $\lambda S_{\mathrm{v}}$ ss, is the product of the average grain intercept length, $\lambda$, and the surface area density of solid-solid boundary $S_{v} s s$. The second is $\lambda S_{v} s v$ where $S_{v} s v$ is the surface area density of solid-vapor interface. The definition of average grain intercept length assumed that there was no pores in the grains. In terms of the experimental results, the solid-solid boundaries at particle necks were counted separately from solid-solid boundaries (grain boundaries) within the particles. Hence, the experimentaly determined dimensionless parameters could be plotted for the grains using the total surface area density of solid-solid interface in the sample, $S_{\mathrm{v}} \mathrm{ssT}$ and the average grain intercept length, $\lambda_{\mathrm{g}}$. The dimensionless parameters could also be plotted for the particles using only the surface area denity of solid-solid interface at particle necks, $S_{\mathrm{v}}{ }^{s S P}$ and the average particle intercept length, $\lambda_{\mathrm{P}}$. This allowed for the comparison to be made with and without the intraparticle structure.

\section{Experimental Procedures}

The composition of the N625 alloy powder is listed in Table I and Table II: 
Table I: Major Constituents

\begin{tabular}{|l|l|l|l|l|l|}
\hline Element & $\mathrm{Ni}$ & $\mathrm{Cr}$ & $\mathrm{Mo}$ & $\mathrm{Nb}$ & $\mathrm{Fe}$ \\
\hline Wt.\% & 64.10 & 21.2 & 8.73 & 3.7 & 0.06 \\
\hline
\end{tabular}

Table II: Minor Constituents

\begin{tabular}{|l|l|l|l|l|l|l|l|l|l|l|}
\hline Element & $\mathrm{C}$ & $\mathrm{Mn}$ & $\mathrm{Si}$ & $\mathrm{P}$ & $\mathrm{S}$ & $\mathrm{O}$ & $\mathrm{N}$ & $\mathrm{Al}$ & $\mathrm{Ti}$ & $\mathrm{Co}$ \\
\hline Wt.\% & 0.01 & $<0.02$ & 0.01 & 0.001 & 0.003 & 0.01 & 0.10 & 0.13 & $<0.01$ & $<0.01$ \\
& & & & & & & & & & \\
\hline
\end{tabular}

Interrupted HIP cycles were performed using the same computer controlled heating and pressurization cycles. The samples used in this study were HIPed at $50 \mathrm{MPa}$ and $845^{\circ} \mathrm{C}$ for times ranging from 62 minutes to 287 minutes. The samples were removed from the mild steel cans and the sections to be used for microscopy were cut from the center of the HIPed samples.

Metallographic sections were electrolytic etched with an aqueous solution of $5 \%$ nitric acid and 5\% glacial acetic acid and examined with a LECO 2001 image analyzer at 500x magnification. Intercepts were then counted automatically for solid-vapor interface, solidsolid interface at particle necks and solid-solid interface inside the particles. This allows for the calculation of the surface area densities for solid-vapor interface, solid-solid interface formed at particle contacts and solid-solid interface inside the particles. There was no evidence of solid-vapor interface inside the particles. However, it was not always possible to distinguish between small grains of the matrix and grain boundary precipitates during the analysis. Therefore the measured surface area density of intraparticle solid-solid interface contained some contribution from the larger grain boundary precipitates as well as the matrix grains. The average grain intercept length, average pore intercept length and average particle intercept length were also calculated with the intercept data. Finally, the area fraction of pores was determined. For each HIP condition the measurements of intercepts and area fraction were conducted on five separate images, selected at random on each sample. The total number of intercepts counted was at least 500 for both the solid-solid and solid-vapor interface. 
Results

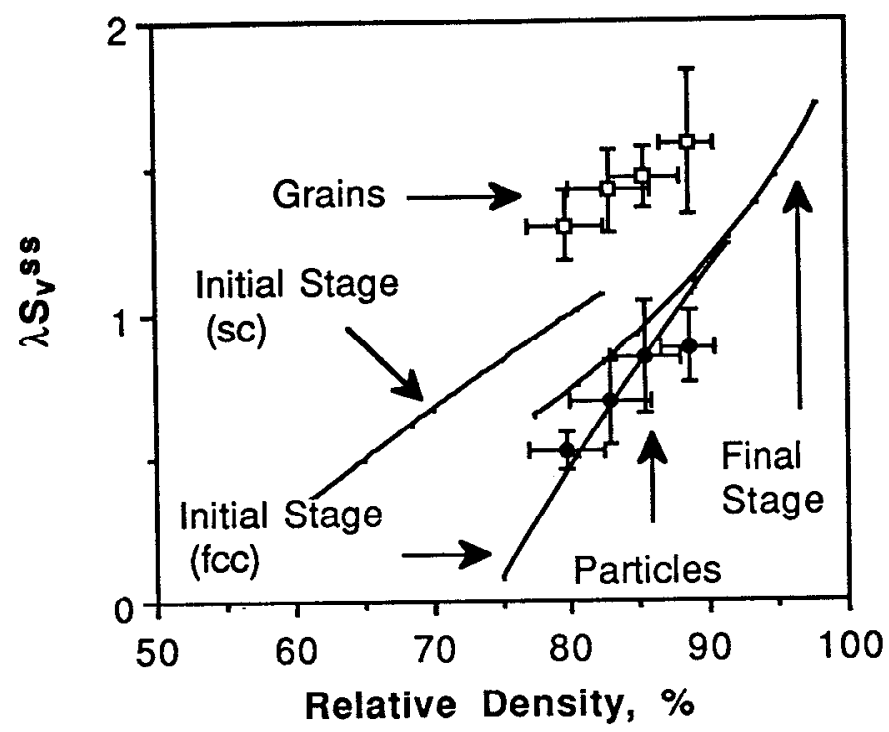

Figure 1: The dimensionless parameter $\lambda S_{\mathbf{v}}$ ss, plotted a function of relative density for initial stage (sc) and (fcc) and final stage. The experimental results $\lambda_{\mathrm{g}} S_{\mathrm{v}} \mathrm{ssT}$ for the "grains" and $\lambda_{\mathrm{p}} S_{\mathrm{v}} \mathrm{ssP}$ for the "particles" are included in the comparison.

Figure 1 shows the effect of density on the dimensionless parameter $\lambda S_{\mathrm{v}}$ ss for some initial stage and final stage model geometries. The results are plotted for initial stage using simple cubic (sc) and face centercd cubic (fcc) packing arrangements of spheres. The models are plotted in the density ranges that satisfy self consistency for the geometry. There were no further attempts to limit the stages of sintering based on other considerations. The models predict an increase in $\lambda S_{\mathbf{v}}$ ss with increasing density. The simple cubic arrangement gave the higher values of $\lambda S_{\mathrm{V}}$ ss when compared with the face centered cubic arrangement. The calculations for final stage, which consist of space filling tetrakiadecahedrons with spherical pores on the vertices, also resulted in values of $\lambda S_{\mathrm{v}}$ ss that increased with density . In the density range $85 \%$ to $90 \%$, the predictions for initial stage ( $\mathrm{fcc}$ ) and final stage gave similar values of $\lambda S_{v}$ ss. Experimental results are also plotted for $\lambda_{g} S_{v} s s T$ where $\lambda_{g}$ is the average grain intercept length and $S_{\mathrm{v}}{ }_{\mathrm{v}} \mathrm{T}$ corresponds to the total solid-solid interface in the material. These results, labeled "grains" in figure 1 , gave values of $\lambda_{\mathrm{g}} S_{\mathrm{v}} \mathrm{ssT}$ which increased from 1.3 at a relative density of $80 \%$ to 1.6 at a relative density $89 \%$. While the observed trend in $\lambda_{\mathrm{g}} \mathrm{S}_{\mathrm{v}} \mathrm{ssT}$ was consistent with the models, the measured values were much higher. None of the model stages gave predictions of $\lambda S_{\mathrm{v}}$ ss above 1.1 in this density range. By separately counting the solid-solid intercepts on particle-particle necks it was 
possible to define an average intercept length for the particles $\lambda_{p}$ and the surface area density of solid-solid contacts at particle necks $S_{v}$ ssP. Thus the dimensionless parameter $\lambda_{\mathrm{p}} S_{\mathrm{v}}$ ssP could be measured. The results are labeled "particles" in figure 1 . These results show much closer agreement with the models, especially initial stage ( $\mathrm{fcc}$ ). All the experimental measurements of $\lambda_{\mathrm{p}} \mathrm{S}_{\mathrm{v}} \mathrm{ssP}$ are within the experimental error of the initial stage (fcc) prediction.

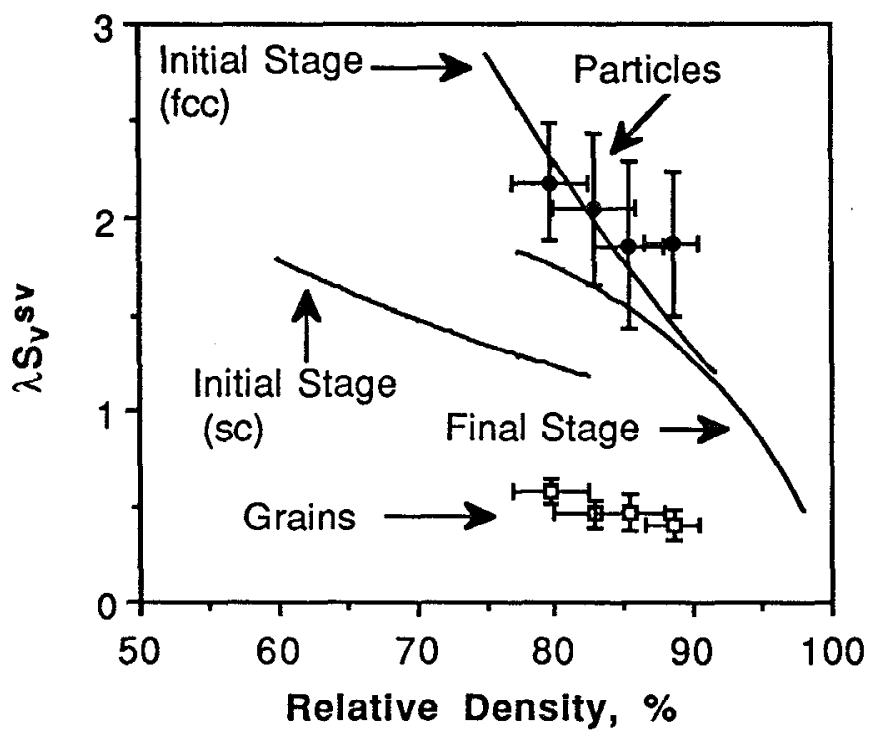

Figure 2: The dimensionless parameter $\lambda S_{\mathrm{v}} \mathrm{sv}$ as a function of density for the model stages and the experimental results, $\lambda_{\mathrm{g}} S_{\mathrm{v}} \mathrm{sv}$ for the "grains" and $\lambda_{\mathrm{p}} S_{\mathrm{v}} \mathrm{sv}$ for the "particles"

Figure 2 shows the predictions of $\lambda S_{\mathrm{v}}$ sv for the initial stage models and the final stage model. All the models showed a decrease in this parameter as the relative density increased. For the initial stage models, the simple cubic packing arrangement gave a lower prediction of $\lambda S_{\mathrm{v}} \mathrm{sv}$ than the fcc arrangement. The final stage model and initial stage fcc model again show similar predictions for $\lambda S_{\mathrm{v}} \mathrm{sv}$ in the density range $85 \%$ to $90 \%$.

Experimental measurements of the average grain intercept length and the total surface area density of solid-vapor boundary allowed for the calculation of $\lambda_{\mathrm{g}} S_{\mathrm{v}} \mathrm{sv}^{\mathrm{sv}}$ for the HIIPed N625 alloy. The experimental values, labeled "grains", show a modest decrease in $\lambda_{\mathrm{g}} \mathrm{S}_{\mathrm{v}} \mathrm{sv}$ from 0.6 at a relative density of $80 \%$ to 0.4 at $89 \%$. These values were much lower than the model prediction in the same density range. The same dimensionless parameter could be 
measured for the "particles" using the average particle intercept length $\lambda_{p}$, and the surface area density of solid-vapor boundary, $S_{v}{ }_{v}{ }^{s v}$. The results for $\lambda_{p} S_{v}{ }^{s v}$ varied between 2.2 at $80 \%$ and 1.7 at $89 \%$. These values were well above the experimental results for the "grains". Again, the results for the "particles" were in good agreement with the initial stage (fcc) model.

\section{Discussion}

The two dimensionless parameters used in this study allow for a direct comparison between the model stages of densification and experimental results. The parameters $\lambda S_{\mathrm{v}}{ }^{s s}$ and $\lambda S_{\mathrm{v}} \mathrm{sv}$ require the measurement of two stereological quantities that have unique values for any microstructure. The average intercept length is a derived length scale based on the surface area density measurements and the assumptions that there are no pores in the grains or the particles. In terms of the model stages, the dimensionless parameters can be calculated directly since they are only a function of relative density for the ordered arrays of spheres and tetrakiadecahedrons used in the model stages.(7) Therefore, a new methodology exists for directly comparing the microstructure evolution of real materials with the evolution of the analytical models commonly used to make dynamic predictions of density as a function of time.

This study made comparisons of the models with experimental determinations of the total surface area density of solid-solid interface $S_{\mathbf{v}}{ }^{s s T}$, the solid-vapor interface $S_{\mathbf{v}}{ }^{s v}$ and the average grain intercept length $\lambda_{\mathrm{g}}$. The results show that the model stages underestimate $\lambda S_{\mathrm{v}}$ ss in comparison with $\lambda_{\mathrm{g}} S_{\mathrm{v}} \mathrm{ssT}$ and overestimate $\lambda S_{\mathrm{v}}{ }^{\mathrm{sv}}$ when compared with $\lambda_{\mathrm{g}} S_{\mathrm{v}} \mathrm{sv}$. This disagreement could be interpreted in terms of the internal grain structure of the particles which is not accounted for by the models. The internal structure would tend to increase $\mathrm{S}_{\mathrm{V}}$ ss and decrease $\mathrm{S}_{\mathrm{V}}{ }^{\mathrm{sv}}$ in comparison with the models and thus account for the observed differences. In this study the image analysis was not able to distinguish between small grains of the matrix and some grain boundary precipitates. Therefore not all the precipitates were removed by image processing. This would tend to increase the difference between the model predictions and the results for the grains.

Some possible geometrical refinements to the models would result in better agreement between the models and the experimental determinations of microstructure. One such refinement would involve adding internal grain structures to the spheres or the tetrakiadecahedrons so that they represent particles and not individual grains. Hence, the pores in the models would represent the interparticle pores. Such pores would be larger 
relative to the grain size than when the spheres or the tetrakiadecahedrons are taken to represent individual grains. The new refined model would increase $S_{\mathrm{v}} \mathrm{ss}$ and decrease $S_{\mathrm{v}} \mathrm{sv}$ in comparison with the original models where the spheres and polyhedra are taken to represent grains. Therefore, better agreement would be expected between the model and the experiment.

The gas atomized N625 alloy powders examined in this investigation are representative of the simplest case of the modified models in which powder compacts are made up of dense, spherical, polycrystalline powder particles. By separately counting the solid-solid intercept at particle necks, the internal structure of the particles could be effectively removed for the comparison with the models. The results in figure 1 and figure 2 showed that this lead to much better agreement with the models in terms of the variation of the dimensionless parameters with relative density. In the density range of the experimental results the agreement between the experimental results for the particles and the initial stage fcc model was very good. This clearly showed that better agreement is obtained if the internal structure of the particles is removed. The problem encountered in distinguishing between small grains and some grain boundary precipitates is also avoided in this approach.

While the results of this study tend to support the refinement of the analytical models of sintering by placing internal grain structure into the spheres or tetrakiadecahedrons, this may not be appropriatc to all cases. There are other refinements that can also give better agreement between the models and experimental results. For example, some of the porosity could be included as vacancies in the arrangement of spheres or tetrakiadecahedrons used to represent the grains. This would also lead to an increase in $\lambda S_{\mathbf{v}}$ ss and a decrease in $\lambda S_{\mathrm{v}}{ }^{\mathrm{sv}}$ such that the model better reflected the trends in the experimental results of $\lambda_{\mathrm{g}} S_{\mathrm{v}}$ ssT and $\lambda_{\mathrm{g}} \mathrm{S}_{\mathrm{v}} \mathrm{sv}$. It may be necessary to conduct quantitative microscopy before choosing an appropriate refinement to the model for a particular type of powder.

It must also be remembered that the refined models will only reflect the real microstructures in terms of the microstructural averages that were used to define the dimensionless parameters used in this study. They are not meant to fully describe the changes in the microstructure. This would require the consideration of particle size distribution and the spatial heterogeneity in the particle packing. Despite this, the addition of an internal grain structures into the model may be a suitable refinement for developing micromechanical models for the HIPing of gas atomized metal powders. 


\section{Conclusions}

Dimensionless parameters were used to directly compare the microstructure evolution of a HIPed gas atomized N625 metal powder with that of the model stages of sintering commonly used in the micromechanical modeling of HIPing. The results show that the models underestimate $\lambda S_{v} s s$ and overestimate $\lambda S_{v}{ }^{s v}$ when the internal structure of the metal particles are included in the measurements. Removal of the internal structure gave much better agreement between the models and the experimental results. This suggests that the geometrical models for the stages of sintering could be refined to include an internal grain structure in the spheres and polyhedra.

\section{Acknowledgments}

The authors wish to acknowledge the financial support of the National Science Foundation through grant No CMS-94-09456.

\section{References}

1. G.H. Gessinger, Powder Metallurgy of Superalloys (Butterworth Monographs in Materials, London, 1984). 19

2. M. Abouaf, J.L. Chenot, G. Raisson and P. Baudin, "Finite Element Simulation of Hot Isostatic Pressing of Metal Powders," J. Numerical Methods in Engineering. 25 (1988) 191-212.

3. A.S. Helle, K.E. Easterling and M.F.Ashby, "Hot-Isostatic Pressing Diagrams: New Developments," Acta Metall. 33 (1985) 2163-2174.

4. R.L. Coble, "Initial Sintering of Alumina and Hematite, J. Am. Ceram. Soc. 41 (1958) 55-62.

5. R.L Coble, "Sintering Crystalline Solids: I Intermediate and Final Stage Models, J. Appl. Phys. 32 (1961) 787-792.

6. E. Arzt, "The Influence of an Increasing Particle Coordination on the Densification of Spherical Powders," Acta Metall. 30 (1982) 1883-1980.

7. W.S. Slaughter, I. Nettleship, M.D. Lehigh and P.P. Tong, "A Quantitative Analysis of the Effect of Geometric Assumptions in Sintering Models," Submitted to Acta Materialia.

8. E.E. Underwood, Quantitative Stereology, (Addison-Wesley, Reading Mass. 1970).

9. F.J. Rizzo and J.J. Conway, J.F. Radavich and J.A. Jones, "Investigation of Nitrogen Atomized PM Alloy 625," Superalloys 718,625.706 and Various Derivatives (Edited by E.A. Loria, TMS 1994) 923-934. 